\title{
Control Inflammatory Effect in Tissue Engineering with Chitosan Nanoparticles, Influence of Sterilization Process
}

\author{
A. Larena ${ }^{1, *}$, D.A. Cáceres ${ }^{1}$ and G. Ramos-Ortiz ${ }^{2}$ \\ ${ }^{I}$ Department of Chemical Industrial Engineering and Environment, ETS de Ingenieros Industriales, Universidad \\ Politécnica de Madrid, José Gutiérrez Abascal, 2 - 28006 Madrid, Spain \\ ${ }^{2}$ Centro de Investigaciones en Óptica, A.P. 1-948, 37000 León, Gto., México
}

\begin{abstract}
Tissue engineering and regenerative medicine are disciplines in constant evolution to provide solutions, to assist and accelerate the regeneration and repairing of defective and damaged tissues. Many repair techniques produce inflammatory effects that difficult the regenerative process. In the present work we present a summary of our experience in using chitosan formulations as cells carrier for bone and cartilage regeneration. We have found that the use of chitosan lead to inflammatory processes in tissular repairing. To overcome this we proposed the introduction of anti-inflammatory products during the tissular regeneration and here we describe the experimental results on the control inflammatory effect in tissue engineering with chitosan nanoparticles under different sterilization process. It was found that chitosan nanoparticles have potential applications for several therapeutical drugs administration.
\end{abstract}

Keywords: Chitosan nanoparticles, inflammatory effect, tissue engineering, regenerative medicine.

\section{INTRODUCTION}

Chitosan (CHI) is a decalcified and desacetylated chitin compound, an abundant natural polymer. Chitin molecule mainly consists in $\mathrm{N}$-acetylglucosamine aminosugar molecules bound by covalent bonds and hydrogen bridges in a straight polymeric chain. Some chitosan biomaterials like membranes, beads, micro and nanoparticles and composites have been reported in literature [1-5]. Chitosan is a non-toxic biodegradable polycationic polymer with low immunogenicity. It has been extensively investigated for formulating carrier and delivery systems for therapeutic macrosolutes [2]. Chitosan is also a very versatile biomaterial that finds applications in process of tissue engineering and regenerative medicine and in wide field of utilities like wound dressing [4], prosthesis substitute [6], controlled drug release [7, 8], post-operatory material and other applications [9] due to its biocompatibility with human cell cultures [10].

In previous works we have reported diverse studies on Chitosan [9-15]. For instance, chitosan viability as osteoblast culture environment and tissular regeneration was proved, though its implantation in rabbits has some incompatibility problems based in inflammatory processes. In those studies it was found that the analysis of pathologic anatomy shows an inflammatory abscess with necrosis and presence of granulomas of external body that implied the presence of neutrophils and necrosis.

Different compounds can be used to enhance the biocompatibility of chitosan. For example, polyethylene

*Address correspondence to this author at the Department of Chemical Industrial Engineering and Environment, ETS de Ingenieros Industriales, Universidad Politécnica de Madrid, José Gutiérrez Abascal, 2 - 28006 Madrid, Spain; E-mail: alarena@etsii.upm.es glycol (PEG) has been widely used for many authors because it could improve the controlled release and biocompatibility characteristics of chitosan biomaterials, with the ability to enhance therapeutic peptides and proteins and its capacity of binding to different functional groups of macromolecules [16, 17] like acetyl and amine chitosan groups. Nevertheless, the PEG content and crosslinking degree has a great effect on the properties of the porous membranes, including pore structure, stability and mechanical properties [18].

Recently some new biological molecules like Tripolyphosphate (TPP) and Genipin [19] have been studied to drug release and implant biocompatible materials. TPP is a nontoxic crosslinking agent which is able to form chitosan beads, micro and nanoparticles. The interaction between chitosan and TPP depends on the $\mathrm{pH}$ of the solution [20]. It is known that these systems produce dermal and mucosal irritation but its effects are less severe to cells than those produced by glutaraldehyde (i.e., Chanda et al. [21]), sodium hydroxide and other reactants traditionally used as crosslinking agents.

On the other hand, some incompatibility problems based in inflammatory processes can be controlled with nonsteroidal anti-inflammatory drugs (NSAIDs). These NSAIDs are widely used in common medical practice and different NSAIDs controlled release methods have been described by means of chitosan (CHI) membranes or $\mathrm{CHI} /$ hydroxyapatite composite usage. Now we can compare several preparation of chitosan under sterilization process to get insight on NSAIDs controlled release. We point out that many researches have tried to obtain nanoparticles with controlled release characteristics but none of them have studied the effect of autoclave sterilization processes on their properties and their possible application to the daily medical practice. 
In particular, we are interested in Acetylsalicylic acid (ASA) and ibuprofen (IBU) as NSAIDs and the NSAIDs kinetic of chitosan nanoparticles and the polyethylene glycol (PEG) influence in its composition.

The structure of this paper is as follows: Section II presents a summary of our previous studies on chitosan formulations for the incorporation and penetration of chondrocytes and osteoblast in rabbits. The formulations for chitosan membranes, culture of chondrocytes and osteoblasts in those membranes and their implantation in rabbits is described as well as the consequences on induced inflammatory processes. Section III presents alternative solutions to the use of chitosan formulations for bone and cartilage regeneration based in use anti-inflammatory controlled release methods by chitosan nanoparticles.

\section{CHITOSAN FORMULATIONS AND PREVIOUS STUDIES IN THE INCORPORATION AND PENE- TRATION OF CHONDROCYTES AND OSTEOBLAST IN RABBITS.}

\section{II.1. Formulations for Chitosan Membranes}

As it is known, for a correct osseous regeneration it is required the utilization of materials comprising an adequate capacity to produce osteoinduction through cellular proliferation. This cellular proliferation will be determined by diverse aspects as the chemical composition of the base material or its structure in nanometric scale, being the most appropriate for the tissular regeneration those materials that get assimilated more effectively to the tissue to be regenerated. The recent advances in polymer science have led to the design of tissue with nanometric precision which allows hosting osteoblasts in its interior at the time that it permits the entrance of external tissue and the progressive degradation of implanted tissue.

When chitosan is used as biomaterial for osseous regeneration, the obtained results are strongly correlated with the chitosan formulation, the control of its porosity and the sterilization method employed. We performed various experiments on the implantation of chitosan membranes in rabbits as the previous step to the implantation of such membranes in humans in order to demonstrate its validity for osseous regeneration. These studies are the continuation of a series of studies that were performed on the osteoblast culture and the sterilization of chitosan membranes.

Used chitosan $(\mathrm{CHI})$ of low molecular weight and the chitosan crab shell were supplied from SIGMA-ALDRICH as well as the glycerol for cellular insect's culture and the lactic acid (85\%) natural. The chitosan PROTOSAN CL 213 was supplied from NovaMatrix. The sodium Tripolyphosphate (98.5\%) utilized was from Advanced Technology \& Industrial Co. Ltd.

To prepare CHI membranes we employed acetic acid AA (1.5\%) and glycerol GLY (5 \%): $0.7 \mathrm{~g}$ of chitosan was dissolved in $20 \mathrm{ml}$ of water with concentration of $1.5 \%$ acetic acid and $1.6 \mathrm{ml}$ of glycerol. The solution was deposited in Petri dish, $9 \mathrm{~cm}$ of diameter, and allowed to be at $23^{\circ} \mathrm{C}$ and relative humidity of approximately $50 \%$ to get a transparent membrane, here after denoted as CHI-AA-GLY. On the other hand, formulations of chitosan-lactic acid- glycerol were also prepared (CHI-AL-GLY) for subcutaneous test in rabbits: formulations of $\mathrm{CHI}$, lactic acid $(0.75 \%)$ and glycerol (5\%) were utilized for subcutaneous toxicological analysis in rabbits to know the viability of acid lactic as a substitute of the acetic acid as a solvent for chitosan. These formulations were processed from flakes of chitosan, and this produced solutions with high viscosity such that magnetic stirring (at low frequencies) for periods of time between 48 and 72 hours (at room temperature) had to be implemented to dissolve completely the chitosan. The mixture was dried in Petri dishes at $23^{\circ} \mathrm{C} \pm 2$ and $50 \%$ of relative humidity during one week to form the membrane of high elasticity and suitable for its implantation. We also used powder of Chitosan with low molecular weight, and with lower grade of deacetilization than the used in the previous formulation for membrane preparation of CHI-AA-GLY and CHI-AL-GLY. In these cases chitosan was dissolved in less than $24 \mathrm{hrs}$ under magnetic stirring (at low frequencies) and the formation of the membrane was three times faster, but its surface was less uniform and tend to lose elasticity making of them not suitable for posterior die cuts. When samples from both formulations were dried in an oven at $100^{\circ} \mathrm{C}$ or in vacuum at room temperature, the membranes formed faster but with less elasticity.

The chitosan membranes were treated with a lyophilization process and the chitosan pore size was controlled; this with the aim of using pored and cross-linked chitosan tissue for the regeneration of bones rich in collagen, or in composition high in hydroxyapatite. It is worth to mention that the lyophilization process in chitosan membranes did not affect the chitosan pore size; therefore, this provides support to the use of pored and cross-linked biopolymer tissues to regenerate bones rich in collagen, or in composition high in hydroxyapatite, adding a differentiation factor as in the fluids of the tissues.

Once samples were prepared, SEM microscopy was used in rabbit bones and the mercury porosimetry in chitosan membranes to select those membranes with the appropriate degree of porosity for the osseous structure to be regenerated. Then, samples were sterilized using an autoclave through the following steps: 1) sterilization with out sample to clean the steam sterilizer at $134^{\circ} \mathrm{C}$ for 44 minutes, 2) chitosan membrane sterilization at $134^{\circ} \mathrm{C}$ for 18 minutes, 3) sterilization with out simple to clean the steam sterilizer at $134^{\circ} \mathrm{C}$ for 44 minutes. The formulation for the subcutaneous test were performed with a sterilizer of gamma rays at $25-\mathrm{kGy}$ in a medical institution of FREMAP.

\section{II.2. Culture of Chondrocytes and Osteoblasts in Chitosan Membranes}

In the culture of chondrocytes, the cells obtained from the cartilage of the femoral condyles after sequential digestion were growth on chitosan membranes in Dulbbecco's Modified Eagle Médium 10938 (DMEM, supplied from Invitrogen Corporation). This DMEM was supplemented with $1 \%$ of piruvic acid, $1 \%$ of solution of penicillin and streptomycin and with $20 \%$ of fetal bovine serum, replacing the medium every five days. The same procedure than in the previous case was followed: when an appropriate number of cells were estimated, chitosan samples were carefully added. The chitosan samples were 
recollected after 2, 7 and 15 days to perform qualitative and quantitative studies about the viable cells in their interior. It was observed an abundant cell number after 5 days. The use of these samples for the implant in rabbits is performed between the second and sixth cellular pass. Similar procedure was followed for the osteoblasts culture: the obtained cells from the rabbit vertebra were cultured by introducing them along with the membranes in the DMEM described above, replacing the medium every 5 days.

\section{II.3. Membrane Implantation in Rabbits and Biocompa- tibility Analysis}

After the preparation and sterilization of different chitosan samples, the membranes were implanted in generated defects in the internal condyles of rabbits knees and subcutaneous tests were performed. The implant biocompatibility was determined by means of anatomopathologic studies, resulting in the following: the experimental membrane implantation showed a high fragility in those membranes with small defects. This suggested that defects of larger size should be generated in future implants, or alternatively, other membrane fabrication should be utilized. This can be done with the use of a proper microtome. To reduce the fragility the content of chitosan can be increased up to $1 \mathrm{~g}$. Alternatively, lyophilization techniques could help to produce pored implants of better flexibility. On the other hand, it was observed that the membranes induce a toxic reaction in the knees tissue with considerable inflammation for the rabbits under study. Under these circumstances, the experiments derived in a series of procedures as described below.

First, preparation and sterilization of the different chitosan formulations, implantation of the membranes in the knee's internal condyles of the rabbits and subcutaneous tests for CHI-AA-GLY and CHI-LA-GLY formulations were performed. To carry out the implants in knees, the rabbits were anesthetized by sedation and the internal condyles were perforated using a milling machine with 5 $\mathrm{mm}$ of diameter and $4 \mathrm{~cm}$ in deep; disks membranes with that diameter were successively inserted until the cavity was filled. In this case the membranes were inserted in one of knees for each rabbits, while in the others knees membranes with culture of osteoblasts were inserted.

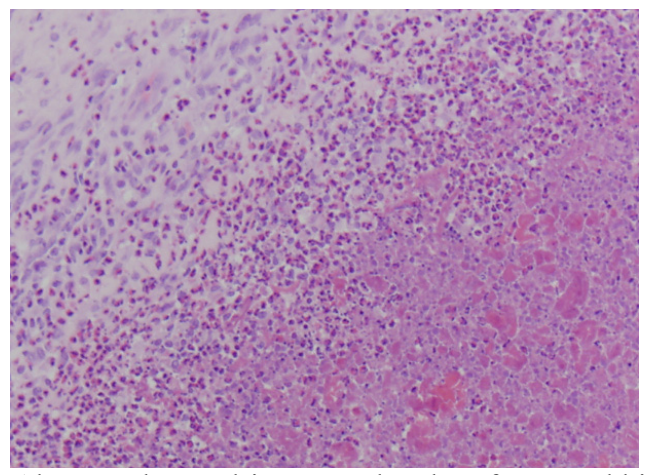

Fig. (1). Abscess observed in a sample taken from a rabbit treated with a subcutaneous test of CHI-AL-GLY.

Both, implantation of CHI-AA-GLY membranes and subcutaneous test with CHI-AL-GLY produced pathology of rejection. In those studies it was found that the analysis showed an inflammatory abscess with necrosis and the presence of granulomas in response to an external body that implied the presence of neutrophils and necrosis. The presence of neutrophils and necrosis implied a severe inflammation as shown in Figs. (1) and (2).

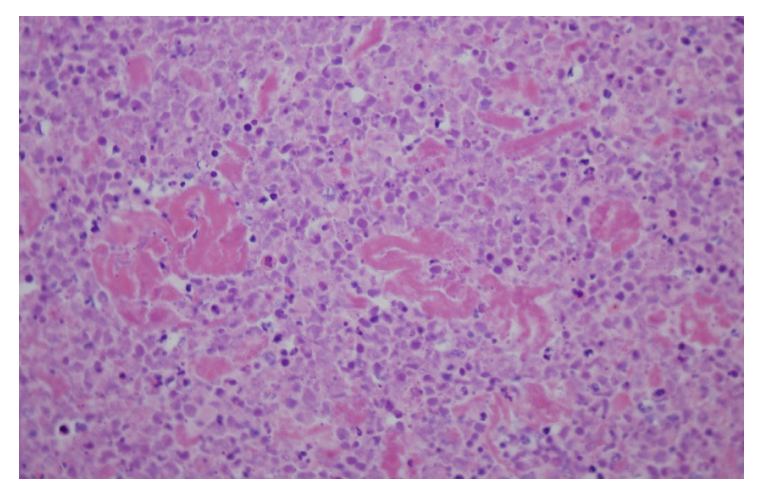

Fig. (2). Necrosis produced in rabbits after the subcutaneous injection of samples of CHI-AL-GLY.

Finally, to corroborate the effect of chitosan concentration on the implants the animals were sacrificed at the intervals of 1,2 and 3 weeks. Then, samples from the rabbits were taken and studied under techniques of microscopy observation. In this case no tissular alterations were observed in the presence of chitosan, neither adverse reactions took place in the animals after surgery during the duration of the experiments.

In conclusion, experiments done with acetic acid composes lead to compatibility problems and toxic reactions. The use of lactic acid as substitute to the acetic acid showed an aggressive reaction as well in the subcutaneous test. To overcome the difficulties above described for the implantation, some experiments were carried out with the introduction of small quantities of anti-inflammatory standard at the moment of the implantation. In this case the implants were performed in rabbit tendons. The samples were introduced in the tendon of Achilles rabbits previously anesthetized. After that, the obtained extremes were united applying chitosan between both extremes and around of it. In the left knee of each rabbit chitosan with concentration A was introduced and in the right knee a concentration B, both with the anti-inflammatory standard. In this case no adverse reaction was observed. These results motivated the search for other formulations with similar potential for the culture of osteoblast and tissular regeneration that eliminates rejection and inflammatory reaction problems observed in previous experiments. This conducted to a proposal of using nanotechnology as a tool to improve the obtained results, in particular, in studying the use of chitosan nanoparticles as carriers of anti-inflammatories with controlled release. This topic is the subject of the following section.

\section{NSAIDS CONTROLLED RELEASE OF CHITOSAN NANOPARTICLES}

\section{III.1. Materials}

Used chitosan $(\mathrm{CHI})$ was chitosan crab shell with $85 \%$ minimum deacetylated supplied from SIGMA-ALDRICH. Sodium tripolyphosphate pentabasic pure (TPP) was 
supplied from FLUKA. PEG 6000 supplied from CARLO ERBA.

\section{III.2. Preparation of CHI Materials}

$\mathrm{CHI}$ membranes formation was as described in section II. More details can be found in Ref. [11]. Briefly, for the preparation of $\mathrm{CHI}$ membranes we followed a modified method to that reported by $\mathrm{Hu}$ et al. [22]. A known amount of CHI was dissolved in $20 \mathrm{ml}$ of water with $1.5 \%$ of acetic acid at room temperature to form different solutions. $1.6 \mathrm{ml}$ of glycerin (5\%) was then added. The solutions was deposited in a $9 \mathrm{~cm}$ of diameter Petri dish and allowed to be at $23^{\circ} \mathrm{C}$ and relative humidity of approximately $50 \%$ to get a transparent membrane.

For preparing membranes loaded with ASA and IBU the method reported by Shu and Zhu [23] was followed with slight modifications. A known amount of drug IBU or ASA $(0.2 \mathrm{gm})$ and $1.6 \mathrm{ml}$ of $5 \% \mathrm{w} / \mathrm{t}$ glycerine were added to $1.5 \%$ w/t CHI solution in aqueous acetic acid $1.5 \% \mathrm{w} / \mathrm{t}$. After solvent evaporation thin membranes were obtained.

To prepare chitosan nanoparticles and NSAID loaded nanoparticles the method proposed by $\mathrm{Xu}$ and $\mathrm{Du}$ was employed [24]. Chitosan dissolution in 1.5\% acetic acid was prepared, and then TPP was added to this dissolution under continuous stirring at $23^{\circ} \mathrm{C}$ and $50 \%$ of relative humidity. This produced nanoparticles which were used to obtain loaded nanoparticles. $0.2 \mathrm{~g}$ of NSAIDs was incorporated to chitosan dissolution before adding the TPP. PEG dissolution was added to some CHI dissolutions before adding the TPP solution. After the nanoparticles preparation, solutions were allowed to stand under refrigeration conditions over night, then the supernatant was eliminated and the sediment was dried in a VACIOTEM SELECTA vacuum oven at $65^{\circ} \mathrm{C}$ with a pressure of 0.8 bar. Table 1 shows the nanoparticle samples composition.

Table 1. Samples Composition of Nanoparticles

\begin{tabular}{|c|c|c|c|c|c|}
\hline $\begin{array}{c}\text { TPP sol. } \\
(\mathbf{m l})\end{array}$ & $\begin{array}{c}\text { CHI sol. 1 } \\
(\mathbf{m l})\end{array}$ & $\begin{array}{c}\text { CHI sol. 2 } \\
(\mathbf{m l})\end{array}$ & $\begin{array}{c}\text { PEG sol. } \\
\mathbf{1}\end{array}$ & $\begin{array}{c}\text { PEG sol. } \\
\mathbf{2}\end{array}$ & $\begin{array}{c}\text { PEG sol. } \\
\mathbf{3}\end{array}$ \\
\hline \hline 48 & 20 & $\mathrm{X}$ & $\mathrm{X}$ & $\mathrm{X}$ & $\mathrm{X}$ \\
\hline 48 & 20 & $\mathrm{X}$ & 20 & $\mathrm{X}$ & $\mathrm{X}$ \\
\hline 48 & $\mathrm{X}$ & 20 & 20 & $\mathrm{X}$ & $\mathrm{X}$ \\
\hline 48 & 20 & $\mathrm{X}$ & $\mathrm{X}$ & 20 & $\mathrm{X}$ \\
\hline 48 & 20 & $\mathrm{X}$ & $\mathrm{X}$ & $\mathrm{X}$ & 20 \\
\hline 7 & 5 & $\mathrm{X}$ & $\mathrm{X}$ & $\mathrm{X}$ & $\mathrm{X}$ \\
\hline 7 & $\mathrm{X}$ & 5 & $\mathrm{X}$ & $\mathrm{X}$ & $\mathrm{X}$ \\
\hline 12 & 5 & $\mathrm{X}$ & $\mathrm{X}$ & $\mathrm{X}$ & $\mathrm{X}$ \\
\hline 12 & $\mathrm{X}$ & 5 & $\mathrm{X}$ & $\mathrm{X}$ & $\mathrm{X}$ \\
\hline 12 & 5 & $\mathrm{X}$ & 5 & $\mathrm{X}$ & $\mathrm{X}$ \\
\hline
\end{tabular}

TPP sol.: $0.1 \mathrm{~g} \mathrm{TPP} / 100 \mathrm{ml}$ deionized water

CHI sol. $1: 0.3 \mathrm{~g} / 100 \mathrm{ml}$ acetic acid $1.5 \%$

CHI sol 2: $2 \mathrm{~g} / 100 \mathrm{ml}$ acetic acid $1.5 \%$

PEG sol. 1: $0.1 \mathrm{~g}$ PEG / $100 \mathrm{ml}$ deionized water

PEG sol. 2: $0.3 \mathrm{~g}$ PEG / $100 \mathrm{ml}$ deionized water

PEG sol. 3: $0.5 \mathrm{~g}$ PEG / $100 \mathrm{ml}$ deionized water

\section{III.3. Sterilization Treatment}

$\mathrm{CHI} / \mathrm{ASA}$ and $\mathrm{CHI} / \mathrm{IBU}$ membranes as well as CHI/ASA and $\mathrm{CHI} / \mathrm{IBU}$ nanoparticles and nanoparticles without NSAIDS were sterilized in a steam sterilizer at $134-138^{\circ} \mathrm{C}$ for 15-20 min.

\section{III.4. Morphology Analysis}

Nanoparticles morphologies were examined using a JEOL, JSM 6400 scanning electron microscopy at $10 \mathrm{kV}$. For observation, the samples were mounted on metal grids using double-sided adhesive tape; the samples were coated with gold by sputtering (BALZERS SCD 004 Sputter Coater) [25].

A JEOL JEM-2000FX Transmission Electron Microscopy (TEM) was also used for the comparison of the different nanoparticles morphologies. Samples were grinded in an agata mortar and dispersed in ethanol using a sonicator (BRANSON 2210 sonicator).

\section{III.5. Drug Release Model of CHI Materials}

According to previously described procedures [11] for controlled release studies, we used $1 \mathrm{~cm}^{2}$ of CHI membranes and $10 \mathrm{mg}$ of nanoparticles loaded with NSAIDS that were introduced in $70 \mathrm{ml}$ deionized water $(\mathrm{pH} \mathrm{7)}$ as release medium at ambient conditions. At time intervals of: $0,5,10$, $20 \mathrm{~min}, 1,2,5,10,24$ and 27 hours, $6 \mathrm{ml}$ of solutions were taken and the drug content was determined by measuring the Uv-vis absorption spectroscopy using a CARY 13E spectrophotometer at the wavelength range $221-222 \mathrm{~nm}$ for ibuprofen [26] and at $296 \mathrm{~nm}$ for O-acetylsalicylic acid [8].

\section{III.6. Results and Discussion}

\section{III.6.1. Sterilization Treatment}

Sterilization treatment could influence the nanoparticles structure and their NSAIDs controlled release. For instance, it was observed in other based CHI/PEG biomaterials that PEG could be degraded because of sterilization treatment when they are treated at $120^{\circ} \mathrm{C}$ for $45 \mathrm{~min}$. Fig. (3) presents the degradation for sterilization treatment in a CHI/PEG sample where a browning process could be observed.

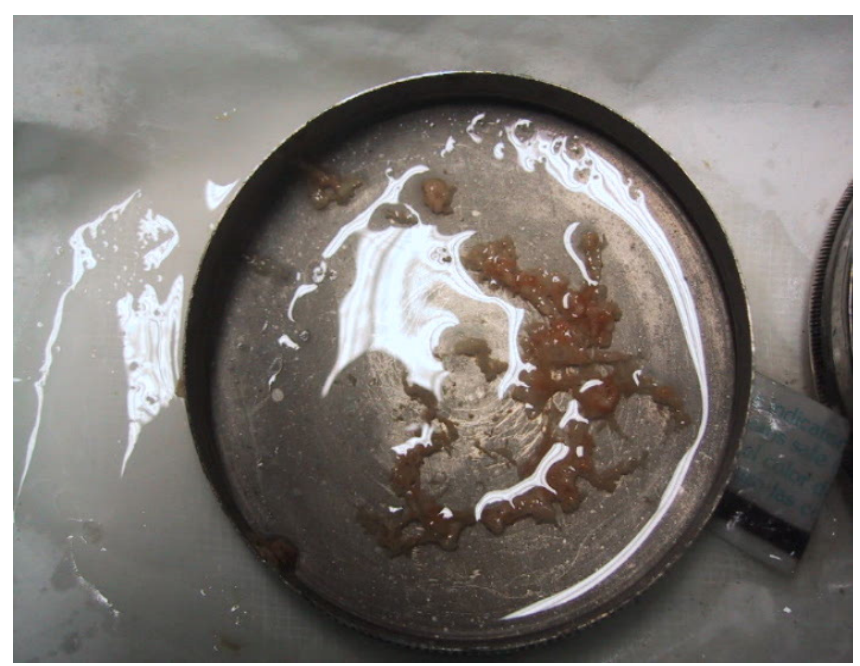

Fig. (3). CHI in $1.5 \%$ acetic acid dissolution loaded with PEG (1g) and submitted to $120^{\circ} \mathrm{C}$ for 45 min sterilization process. 


\section{III.6.2. Morphology Observations}

Figs. (4), (5) and (6) show the differences observed by SEM microscopy between TPP/CHI nanoparticles and TPP/CHI nanoparticles loaded with PEG for the same concentration of TPP and CHI. These results are in agreement with those results observed by TEM microscopy by $\mathrm{Xu}$ and $\mathrm{Du}[24]$ and Shu and Zhu [25].

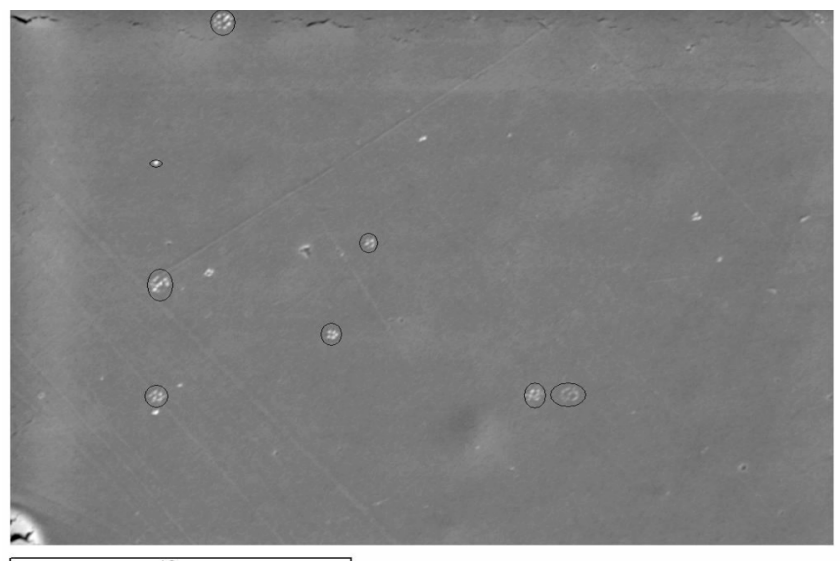

Fig. (4). SEM micrograph of TPP/CHI nanoparticles (CHI 0.3 $\mathrm{g} / 100 \mathrm{ml}$ and TPP $0.1 \mathrm{~g} / 100 \mathrm{ml}$ ). The line for the scale denotes 10 $\mu \mathrm{m}$.

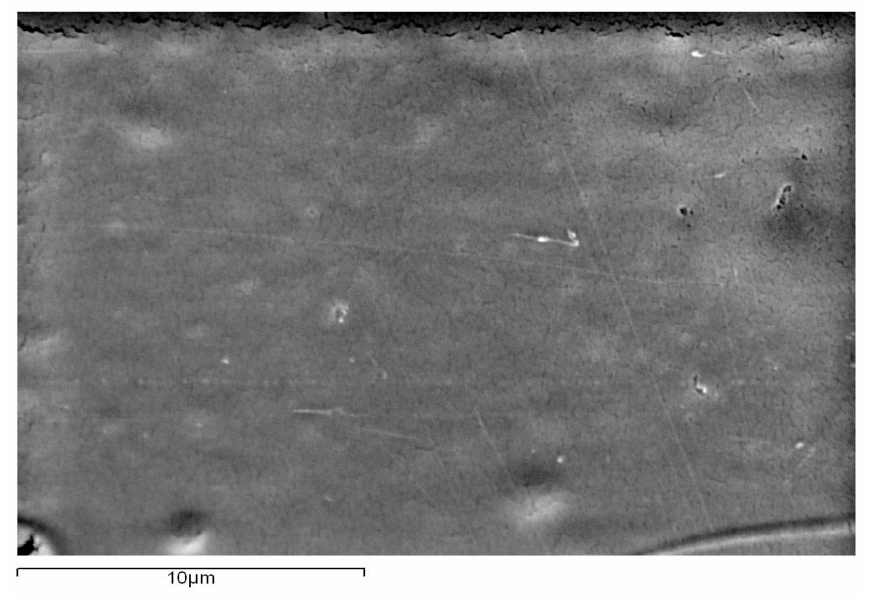

Fig. (5). SEM micrograph of TPP/CHI nanoparticles loaded with PEG (CHI $0.3 \mathrm{~g} / 100 \mathrm{ml}$, TPP $0.1 \mathrm{~g} / 100 \mathrm{ml}$ and PEG $0.1 \mathrm{~g} / 100 \mathrm{ml}$ ). The line for the scale denotes $10 \mu \mathrm{m}$.

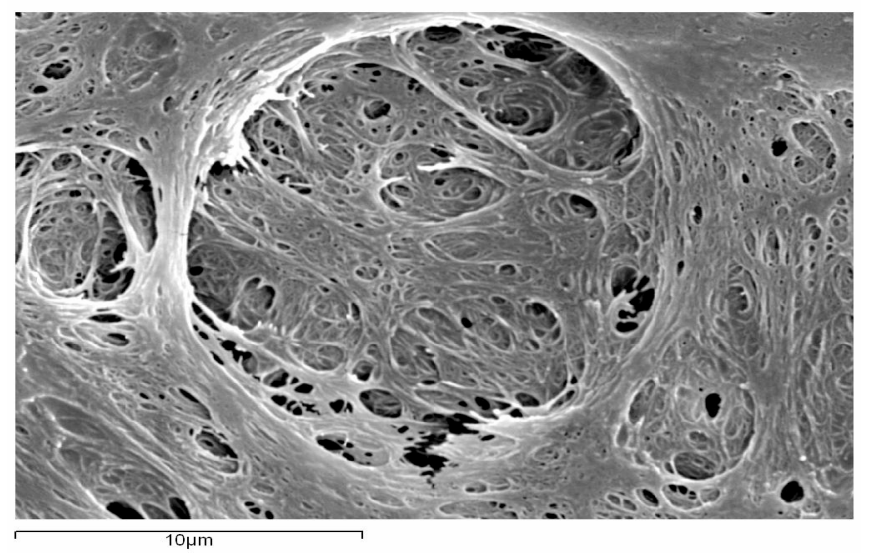

Fig. (6). SEM micrograph of TPP/CHI nanoparticles loaded with PEG (CHI $0.3 \mathrm{~g} / 100 \mathrm{ml}$, TPP $0.1 \mathrm{~g} / 100 \mathrm{ml}$ and PEG $0.5 \mathrm{~g} / 100 \mathrm{ml}$ ). The line for the scale denotes $10 \mu \mathrm{m}$.
It is observed that CHI nanoparticles loaded with $0.5 \mathrm{~g} / 100$ $\mathrm{ml}$ of PEG seem to be "fluffy" [22]. On the other hand, the nanoparticles formed with CHI $2 \mathrm{~g} / 100 \mathrm{ml}$ and $1.5 \%$ of acetic acid present a minor difference between supernatant and sediment; then it is more difficult to separate both of them because of the higher viscosity of the chitosan solution compared with the case of nanoparticles formed with $\mathrm{CHI}$ $0.3 \mathrm{~g} / 100 \mathrm{ml}$ and with $1.5 \%$ of acetic acid.

It is demonstrated that PEG improves the biocompatibility of chitosan films. However additional PEG could deteriorate the mechanical properties, and the biocompatibility; additionally, it can promote uncontrolled cell growth and its proliferation in chitosan films [27] and thus, into nanoparticles. So PEG concentration could be considered as a main factor in controlled release formulations, nanoparticles structure and mechanical properties. We also suppose that PEG concentration could influence the nanoparticles chitosan matrix as it can be observed in Fig. (5) and Fig. (6) in relation with Fig. (4). In Fig. (5) some micro and nanoparticles could be observed. We can consider that amine groups are the usual sites of PEGs linking in the case of polypeptides but this linking depends on $\mathrm{pH}$ [16]. These assertions are in concordance with the $\mathrm{Xu}$ and $\mathrm{Du}$ [24] discussion about the incorporation of PEG in gel system for a bonding between amino hydrogen of chitosan and oxygen atom of PEG.

Figs. (4) and (7) show the differences between TPP/CHI nanoparticles without PEG under different $\mathrm{CHI}$ concentrations. The more the $\mathrm{CHI}$ content the more granular the sample seems, this is because the components distribution depends on the $\mathrm{CHI}$ content.

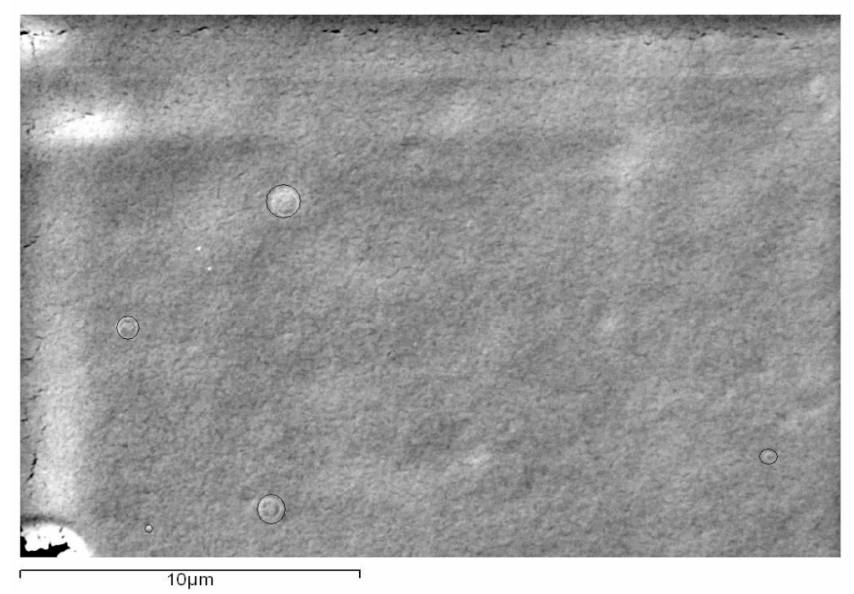

Fig. (7). SEM micrograph of TPP/CHI nanoparticles (CHI $2 \mathrm{~g} / 100$ $\mathrm{ml}$ and TPP $0.1 \mathrm{~g} / 100 \mathrm{ml}$ ). The line for the scale denotes $10 \mu \mathrm{m}$.

SEM micrographs of TPP/CHI nanoparticles loaded with ASA and IBU are represented in Figs. (8), (9), (10) and (11). A change in membrane-nanoparticle morphology could be due to the sterilization process. However heterogeneity of the materials, and the ASA crystallinity could be other causes of the different presentation. In Fig. (9) a porous structure could be induced by the dryness process under vacuum at approx. $60^{\circ} \mathrm{C}$ (see materials and methods) and the sterilization process in which the water and solvent go out the material creating heterogeneous cavities and hollows. Figs. (10) and (11) show the differences observed in 
nanoparticles matrix loaded with IBU due to sterilization. Morphology seems to be different comparing with nanoparticles matrix loaded with ASA.

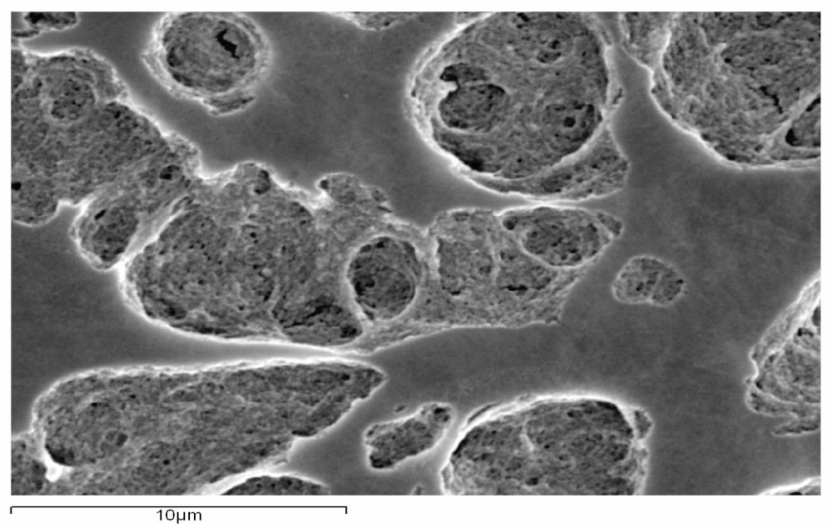

Fig. (8). SEM micrograph of TPP/CHI nanoparticles loaded with ASA (CHI $0.3 \mathrm{~g} / 100 \mathrm{ml}$, TPP $0.1 \mathrm{~g} / 100 \mathrm{ml}$, PEG $0.1 \mathrm{~g} / 100 \mathrm{ml}$ and ASA $0.2 \mathrm{~g})$. The line for the scale denotes $10 \mu \mathrm{m}$.

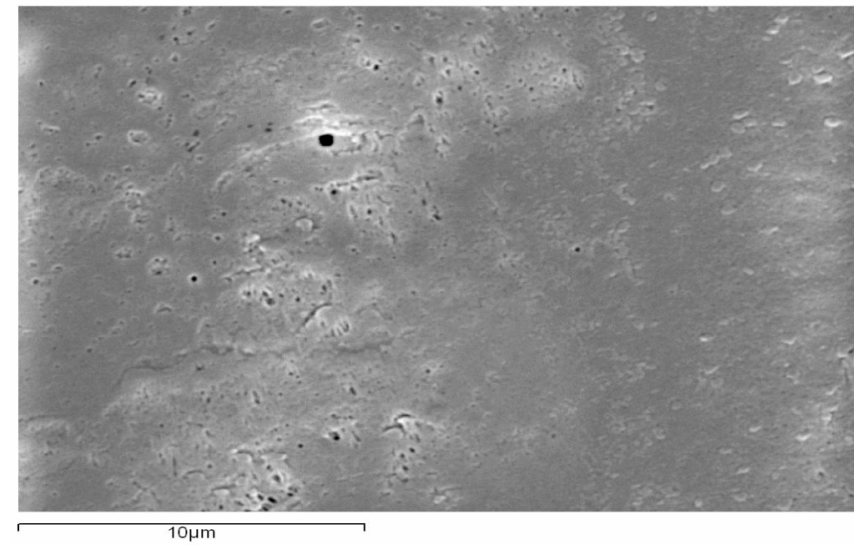

Fig. (9). SEM micrograph of TPP/CHI nanoparticles loaded with ASA and submitted to a sterilization process (CHI $0.3 \mathrm{~g} / 100 \mathrm{ml}$, TPP $0.1 \mathrm{~g} / 100 \mathrm{ml}$, PEG $0.1 \mathrm{~g} / 100 \mathrm{ml}$ and ASA $0.2 \mathrm{~g}$ ). The line for the scale denotes $10 \mu \mathrm{m}$.

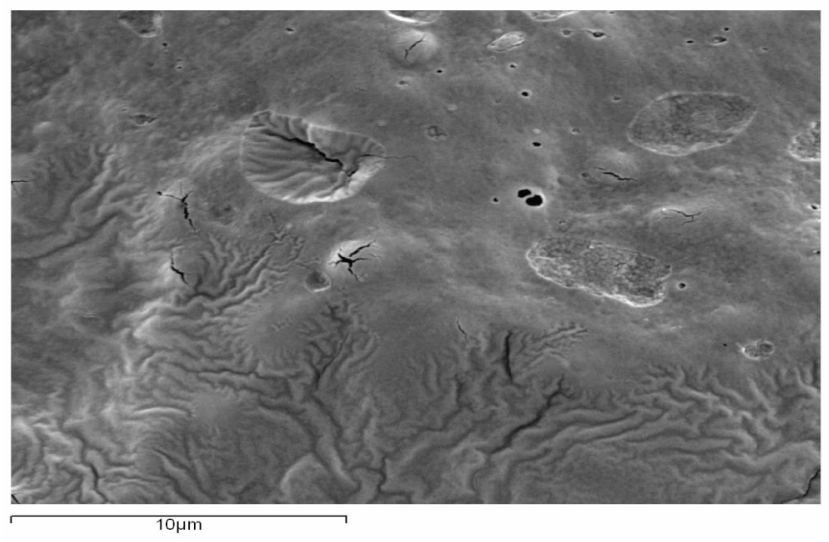

Fig. (10). SEM micrograph of TPP/CHI nanoparticles loaded with IBU (CHI $0.3 \mathrm{~g} / 100 \mathrm{ml}$, TPP $0.1 \mathrm{~g} / 100 \mathrm{ml}$, PEG $0.1 \mathrm{~g} / 100 \mathrm{ml}$ and IBU $0.2 \mathrm{~g})$. The line for the scale denotes $10 \mu \mathrm{m}$.

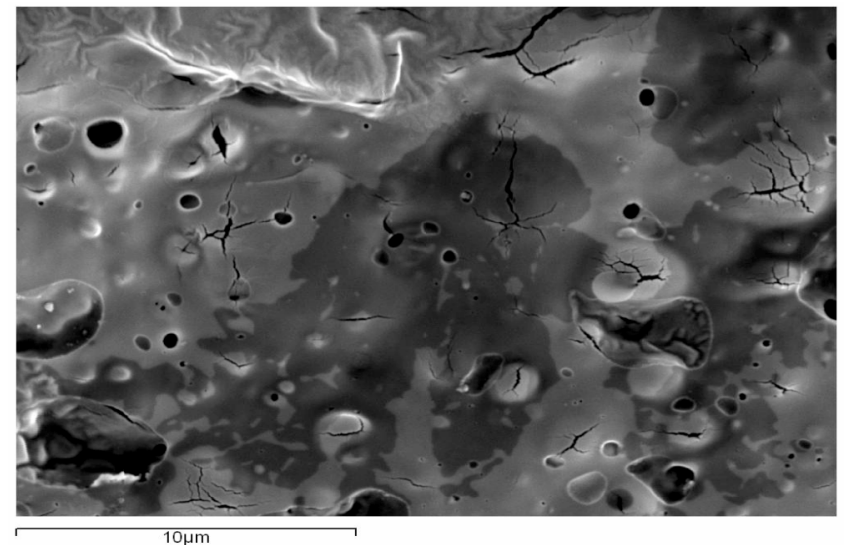

Fig. (11). SEM micrograph of TPP/CHI nanoparticles loaded with IBU and submitted to a sterilization process (CHI $0.3 \mathrm{~g} / 100 \mathrm{ml}$, TPP $0.1 \mathrm{~g} / 100 \mathrm{ml}$, PEG $0.1 \mathrm{~g} / 100 \mathrm{ml}$ and IBU $0.2 \mathrm{~g}$ ). The line for the scale denotes $10 \mu \mathrm{m}$.

TEM micrographs (see Fig. (12)), on the other hand, showed globular structures inside of another structure possibly representing enhancing TPP/CHI nanoparticles. Two kinds of nanoparticles morphologies were observed: a globular form and an angular form. These nanoparticles vary in sizes and morphology and it could represent differences in both compositions. Angular forms could be conditioned by an increase of $\mathrm{CHI}$ concentration but note that $\mathrm{CHI}$ is not really dissolved in ethanol (see Materials and Methods, Morphology observation). On the other hand CHI seems more dissolved, TPP and PEG appears more concentrated in the globular form. Thus, it is convenient to form nanoparticles to take into account the method of preparation and observation (TEM needs a very little quantity of samples comparing with SEM sample method of preparation), possible contamina-tions and the heterogeneity in the composition.
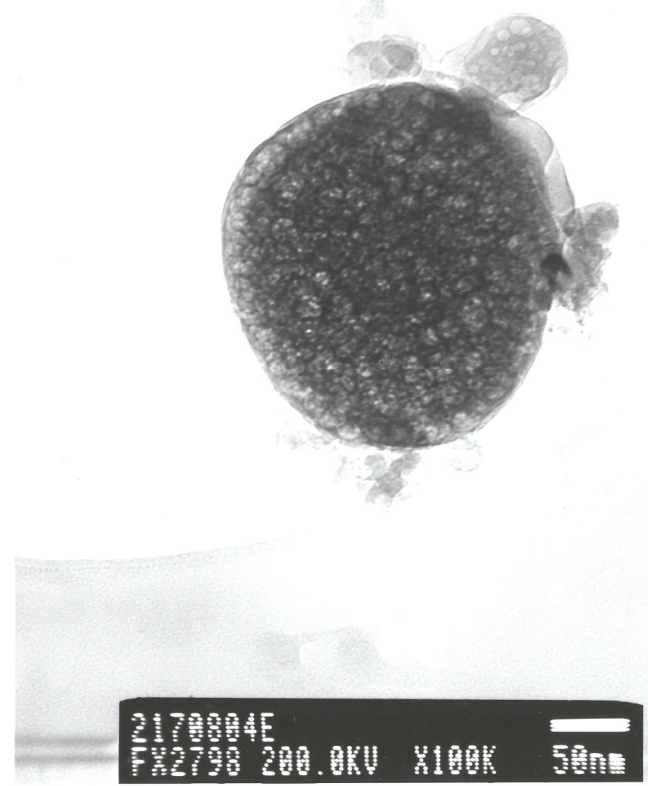

Fig. (12). TEM micrograph of TPP/CHI nanoparticles (CHI 0.3 $\mathrm{g} / 100 \mathrm{ml}$, TPP $0.1 \mathrm{~g} / 100 \mathrm{~m}$, PEG $0.1 \mathrm{~g} / 100 \mathrm{ml}$ ). 


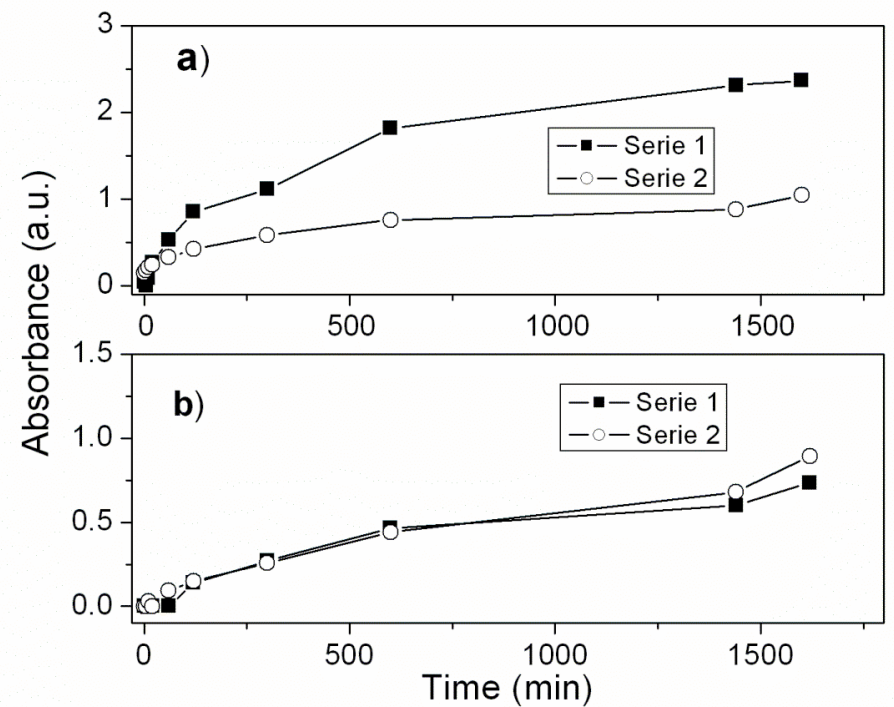

Fig. (13). a) ASA controlled release in deionized water as medium. CHI/ASA membrane. Wavelength $296 \mathrm{~nm}$. b) IBU controlled release in deionized water as medium. CHI/IBU membrane. Wavelength $221 \mathrm{~nm}$. Serie 1: without sterilization treatment. Serie 2: with autoclave sterilization treatment.

\section{III.6.3. Drug Release Model of CHI Materials}

Fig. (13) shows NSAIDS release CHI membranes at ambient conditions [20]. It can be appreciated in Fig. (13b) that sterilization process seems no have influence in IBU controlled release for $\mathrm{CHI} / \mathrm{IBU}$ membranes. However Fig. (13a) shows a difference in controlled release between CHI/ASA membrane submitted to the sterilization and CHI/ASA membranes not submitted. This can be explained for the loss of water in membranes that in this case affects to the membrane structure and the heterogeneity of membrane; ASA do not dissolve as well as IBU.

Fig. (14) shows the controlled release of chitosan nanoparticles loaded with NSAID. Their behavior seems different compared with the membranes (presented in Fig. 13) due to their different morphology. Membranes are more resistant and then in time 0 min no NSAIDs are released. However in the nanoparticles matrix an initial NSAID release is observed for IBU and ASA. ASA release is produced at the beginning of the experiment (time $0 \mathrm{~min}$ ) and its absorbance values remains almost constant during the time (Fig. 14a). It could be explained because of lack of enhancement between ASA and nanoparticles matrix. However Fig. 14b shows other behavior for IBU. It is released during all the experiment, being retained or encapsulated by the nanoparticles matrix although a first release in time $0 \mathrm{~min}$ is also observed. Sterilization process seems no have effect in nanoparticles matrix loaded with ASA unlike the case of nanoparticles-IBU Fig. (14b). SEM micrographs Figs. (8-11) show very different structures for IBU and ASA nanoparticles supports submitted or not to a sterilization process in concordance with controlled release results.

We can conclude that the sterilization process seems no have effect in IBU controlled release for $\mathrm{CHI} / \mathrm{IBU}$ membranes unlike CHI/ASA membranes due to the loss of

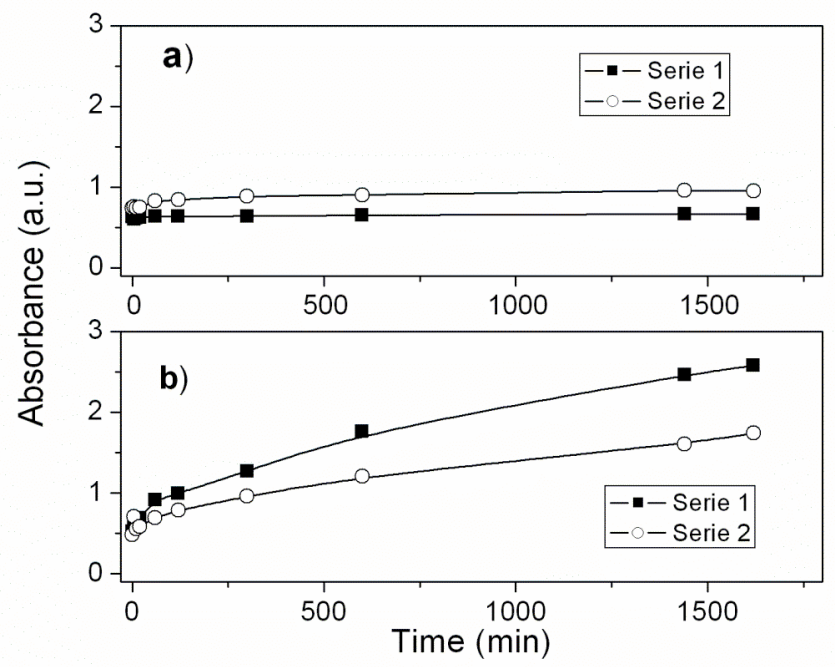

Fig (14). a) ASA controlled release in deionized water as medium. CHI/ASA nanoparticles. Wavelength $296 \mathrm{~nm}$. b) IBU controlled release in deionized water as medium. CHI/IBU nanoparticles. Wavelength $221 \mathrm{~nm}$. Serie 1: without sterilization treatment. Serie 2: with autoclave sterilization treatment. 
water that affects the structure. Nanoparticles have a different behavior. In nanoparticles loaded with ASA steam sterilization have no effect but in microparticles-IBU sterilization has effects due to a very different structure in microparticles matrix. These results show that structure is a very important factor in NSAID release.

\section{REFERENCES}

[1] Chen F, Wang ZC, Lin CJ. Preparation and characterization of nano-sized hydroxyapatite particles and hydroxyapatite/chitosan nano-composite for use in biomedical materials. Mater Lett 2002; 57(4): 858-61.

[2] Janes KA, Calvo P, Alonso MJ. Polysaccharide colloidal particles as delivery systems for macromolecules. Adv Drug Deliv Rev 2001; 47(1): 83-97.

[3] Banerjee T, Mitra S, Singh KA, Sharma RK, Maitra A. Preparation, characterization and biodistribution of ultrafine chitosan nanoparticles. Int J Pharm 2002; 243(1-2): 93-105.

[4] Mi FL, Shyu SS, Wu YB, Lee ST, Shyong JY, Huang RN. Fabrication and characterization of a sponge-like asymmetric chitosan membrane as a wound dressing. Biomaterials 2001; 22 (2): 165-73.

[5] Shi XY, Tan TW. Preparation of chitosan/ethylcellulose complex microcapsule and its application in controlled release of Vitamin $\mathrm{D}_{2}$. Biomaterials 2002; 23(23): 4469-73.

[6] Muzzarelli RAA, Biagini G, DeBenedittis A, Mengucci P, Majni G, Tosi G. Chitosan-oxychitin coatings for prosthetic materials. Carbohydr Polym 2001; 45 (1): 35-41.

[7] Gupta KC, Ravi Kuma MNV. Drug release behavior of beads and microgranules of chitosan. Biomaterials 2000; 21 (11): 1115-19.

[8] Puttipipathachorn S, Nunthanid J, Yamamoto K, Peck GE. Drug physical state and drug-polymer interaction on drug release from chitosan matrix films. J Control Release 2001; 75(1-2): 143-53.

[9] Larena A, Cáceres DA. A review of spectroscopic methods and others for measuring the chitosan degree of deacetilation and its influence in some properties. Trends Appl Spectrosc 2005; 5(1): 247-57.

[10] Larena A, Cáceres DA, De la Piedra C, et al. Estudios de quitosano como portador de osteoblastos en cultivo. Patología del Aparato Locomotor de la Fundación Mapfre Medicina 2004; 2(3): 199-204.

[11] Larena A, Cáceres DA, Vicario C, Fuentes A. Release of a chitosan-hydroxyapatite composite loaded with ibuprofen and acetyl-salicylic acid submitted to different sterilization treatments. Appl Surf Sci 2004; 238(1-4): 518-22.

[12] Larena A, Caceres D. Variability between chitosan membrane surface characteristics as function of its composition and environmental conditions. Appl Surf Sci 2004; 238(1-4): 273-77.
[13] Larena A, Caceres DA, de la Piedra C, et al. Soluciones alternativas al uso de formulaciones de quitosano como portadores de osteoblastos para regeneración ósea. Patología del aparato locomotor Fundación Mapfre-Medicina 2005: 2 (3): 199-204.

[14] Larena A, Caceres DA, de la Piedra C, et al. Preparacion de biomateriales basados en quitosano y estudios como portadores de osteoblastos en cultivo. Patología del aparato locomotor Fundación Mapfre-Medicina 2005; 3 (1): 55-63.

[15] Larena A, Tur A. Lopez-Oliva F, et al. Aproximación nanotecnológica a la regeneración ósea. Patología del aparato locomotor Fundación Mapfre-Medicina 2006; 4 (3): 208-14.

[16] Veronese FM. Peptide and protein PEGylation: a review of problems and solutions. Biomaterials 2001; 22(5): 405-17.

[17] Tirelli N, Lutolf MP, Napoli A, Hubbell JA. Poly(ethylene glycol) block copolymers. Rev Mol Biotechnol 2002; 90(2): 3-15.

[18] Zeng M, Fang Z. Preparation of sub-micrometer porous membrane from chitosan/polyethylene glycol semi-IPN. J Membr Sci 2004; 245(1-2): 95-102.

[19] Mi FL, Tan YC, Liang HF, Sung HW. In vivo biocompatibility and degradability of a novel injectable-chitosan-based implant. Biomaterials 2002; 23(1): 181-91.

[20] Ko JA, Park HJ, Hwang SJ, Park JB, Lee JS. Preparation and characterization of chitosan microparticles intended for controlled drug delivery. Int J Pharm 2002; 249(1-2): 165-74.

[21] Chanda J, Kuribayashi R, Abe T. Use of the glutaraldehydechitosantreated porcine pericardium as a pericardial substitute. Biomaterials 1996; 17 (11): 1087-91.

[22] Hu Q, Fang Z, Zhao Y, Xu C, A new method to prepare chitosan membrane as a biomedical material. Chin J Polym Sci 2001; 19(5): 467-70.

[23] Shu XZ, Zhu KJ. The influence of multivalent phosphate structure on the properties of ionically cross-linked chitosan films for controlled drug release. Eur J Pharm Biopharm 2002; 54(2): 23543.

[24] Xu Y, Du Y. Effect of molecular structure of chitosan on protein delivery properties of chitosan nanoparticles. Int J Pharm 2003; 250(1): 215-26.

[25] Shu XZ, Zhu KJ. Controlled drug release properties of ionically cross-linked chitosan beads: the influence of anion structure. Int $J$ Pharm 2002; 233(1-2): 217-25.

[26] Liu XZ, Fang ZL. Sequential-injection system for drug-dissolution studies of ibuprofen tablets and sustained-release formulations. Anal Chim Acta 1998; 358(2): 103-10.

[27] Zhang M, Li XH, Gong YD, Zhao NM, Zhang XF. Properties and biocompatibility of chitosan films modified by blending with PEG. Biomaterials 2002; 23(13): 2641-48.

Received: April 03, 2009

Revised: May 15, 2009

Accepted: June 03, 2009

(C) Larena et al.; Licensee Bentham Open.

This is an open access article licensed under the terms of the Creative Commons Attribution Non-Commercial License (http://creativecommons.org/licenses/by$\mathrm{nc} / 3.0 /$ ), which permits unrestricted, non-commercial use, distribution and reproduction in any medium, provided the work is properly cited. 